\title{
Présentation d'une méthode simple d'estimation de la contribution des réserves pour le remplissage des grains chez le maïs
}

\author{
F Ruget \\ INRA, station de bioclimatologie, 78850 Thiverval-Grignon, France
}

(Reçu le 31 octobre 1990; accepté le 11 avril 1991)

\begin{abstract}
Résumé - L'intervention de réserves dans le remplissage des grains, quoique connue, est généralement mise en évidence à partir de méthodes lourdes, qui conduisent à diminuer la fréquence des observations ou le nombre de traitements. Or, la datation et la quantification des interventions de réserves, indispensables pour une bonne modélisation de la gestion des réserves, nécessitent des mesures nombreuses. Le présent travail propose une estimation des réserves utilisées pendant le remplissage des grains, à partir de la différence entre croissance des grains et croissance de la plante entière. Cette dernière est estimée grâce au rayonnement intercepté par la culture. L'exemple trajté ici est celui du maïs, mais pourrait s'adapter à toute autre culture où un organe (grains ou tubercules) est rempli de façon régulière, avec appel aux réserves temporaires en cas d'insuffisance des fournitures directes d'assimilats.
\end{abstract}

utilisation de réserves $/$ Zea mays = maïs / interception du rayonnement

Summary - An easy method to quantify reserve use in corn grain filling. The use of storage carbohydrates for grain filling has been known for a long time. However, its quantification by carbohydrate concentration prevents frequent measurements from being made. Use of reserves is measured from the difference between grain growth and whole plant growth. As respiratory losses are very small compared to dry matter decrease, they are not taken into account. Figure 1 shows the evolution of whole plant and grain dry matter during grain filling. For each observation period, reserve use between 2 measurement dates is negatively correlated with whole plant growth (fig 2). Reserve use increases with time for all genotypes (fig 3). We propose an easy method to estimate reserve use based on the difference between actual grain growth and maximal dry matter production (estimated from crop light interception). The results are in good agreement with the measurements except for points corresponding to early stages of grain filling (fig 4). These points correspond to high conversion efficiency (fig 5) at the beginning of grain filling (as has already been reported in the literature). This method allows the frequent quantification of reserve use from grain growth, leaf area index and global radiation measurements. It can be used for maize or other plants for which the storage organ (grain or tuber) is the only one to grow towards the end of the life cycle.

reserve use / Zea mays = maize / crop light interception

\section{INTRODUCTION}

Le fait que des réserves temporaires interviennent lors du remplissage des grains chez le maïs est connu de longue date (Daynard et al, 1969; Derieux, 1978; Varlet Grancher, 1982). C'est grâce à l'intervention de réserves que le remplissage des grains peut être aussi régulier en conditions naturelles (Johnson et Tanner, 1972; Egharevba et al, 1976; Kiniry et Ritchie, 1985), même si l'offre en assimilats est faible, par exemple suite à un effeuillage (Jones et Simmons, 1983). Cette régularité du remplis- sage est utilisée en modélisation de la production du maïs, dans CERES-Maize (Jones et Kiniry, 1986) : les réserves servent à satisfaire la demande des grains lorsque l'assimilation directe est insuffisante.

La caractérisation précise de l'utilisation des réserves, mais surtout les lois de répartition qui régissent la mise en réserves, ne sont pas bien connues. Or, la quantité de réserves présentes est particulièrement importante dans les conditions de culture où les quantités d'assimilats disponibles pour le remplissage des grains peuvent limiter le rendement (forte densité de culture, gé- 
notypes tardifs, etc), c'est-à-dire où la croissance des grains est ralentie (voire arrêtée) par l'épuisement des réserves (Jones et Simmons, 1983).

L'intervention de réserves est généralement mise en évidence par des bilans de matière sèche par organe, des suivis de teneurs en sucres des tiges, principal organe fournisseur de réserves (Daynard et al, 1969; Iwata, 1975; Jones et Simmons, 1983; Barnett et Pearce, 1983) ou des suivis de concentration en ${ }^{13} \mathrm{C}$ après marquage (Cliquet, 1989). Ces dernières méthodes fournissent beaucoup d'informations sur l'attribution puis la remobilisation du carbone, mais elles sont lourdes et ne permettent pas les caractérisations nombreuses que nécessitent, par exemple, les comparaisons entre plusieurs génotypes ou traitements : une méthode plus maniable semble donc utile.

Le fait que les grains soient les seuls organes en croissance permet d'attribuer aux grains tous les assimilats nouveaux. Si la croissance des grains est supérieure à celle de la plante entière, c'est qu'il y a réutilisation d'assimilats provenant d'autres organes, que le bilan de croissance de la plante soit positif ou négatif. La différence entre croissance des grains et croissance de la plante entière fournit une mesure de l'utilisation des réserves. Cette mesure fournit une valeur surestimée, puisqu'elle ne tient pas compte des pertes respiratoires de l'ensemble de la plante. L'impact de cette hypothèse sera discuté ultérieurement.

Par ailleurs, une estimation de l'utilisation des réserves peut être faite par la différence entre la croissance des grains mesurée (qui constitue la demande) et la croissance possible du couvert (l'offre) estimée à partir du rayonnement intercepté par la culture. La méthode proposée permet l'estimation de la quantité de réserves utilisées pendant une période, à partir de la mesure de la croissance des grains et celle du rayonnement intercepté pendant cette période. Elle ne prétend en aucun cas fournir une mesure des réserves présentes, mais seulement estimer leurs variations.

\section{MATÉRIEL ET MÉTHODES}

Des cultures ont été réalisées à plusieurs densités afin que les plantes aient des disponibilités individuelles en rayonnement différentes, donc des niveaux de mise en réserves, de demande en assimilats (nombre de grains) et de remobilisations différents (Ruget, 1989).

\section{Génotypes et conditions de culture}

Les cultures ont été réalisées à Mons-en-Chaussée (Somme, $50^{\circ} \mathrm{N}, 3^{\circ} \mathrm{E}$ ), dans le Nord de la France, en 1984. Les conditions détaillées de culture ont déjà été décrites (Ruget, 1989). Deux génotypes ont été cultivés :

- F7XF2, génotype très précoce, hybride simple entre 2 lignées très employées dans les génotypes adaptés au Nord de l'Europe;

- Dea, génotype demi-précoce en zone limite de culture dans le Nord de la France.

\section{Densités de culture}

Les cultures ont été réalisées à plusieurs densités :

- une, un peu inférieure à la normale de la région ( $d$, environ 6 plantes. $\mathrm{m}^{-2}$ );

- une autre, environ le double $(D=2 d)$;

- une troisième, forte au début, éclaircie quelques j avant la floraison pour revenir à la densité faible (é $=$ $D$ puis $d$ ).

\section{Mesures biologiques et données climatiques}

La croissance en matière sèche du couvert est caractérisée à partir de prélèvements de 6 plantes faits dans chacune des 3 répétitions, chaque semaine, de la floraison à la récolte. Pour alléger les mesures, les plantes ont été pesées par lots de 3 pendant une bonne partie de la culture, puis individuellement avec séparation des grains, pour le dernier mois.

Les indices foliaires nécessaires à l'estimation du rayonnement intercepté par la culture sont calculés à partir de densités de plantes et de surfaces foliaires à la floraison et de comptages de nombres de feuilles vertes. Les surfaces foliaires sont calculées à partir des longueurs et largeurs mesurées feuille par feuille à la floraison (Surface $=$ longueur $\times$ largeur $\times 0,75$; Bonhomme et al, 1982) sur des lots de 30 plantes. Les indices foliaires sont estimés comme décroissant linéairement jusqu'à la date d'observation du nombre de feuilles vertes (environ $3 / 4$ de feuilles encore vertes le jour 282), puis décroissant linéairement jusqu'à zéro pour la récolte de Dea (jour 296).

Le rayonnement global utilisé est celui de la station météorologique la plus proche (Saint-Quentin (Aisne), à $10 \mathrm{~km}$ du site expérimental).

L'utilisation de réserves, mesurée à partir de bilans de matière sèche de grains et de plante entière, est surestimée de la respiration des organes non-grain. Celle-ci, d'après la bibliographie et les décroissances de plante mesurées en fin de cycle (donc hors photosynthèse), est de l'ordre de $0,1 \%$ du poids des organes considérés, soit de l'ordre de $0,1 \mathrm{g.j} \mathrm{j}^{-1}$, donc moins d' $1 \mathrm{~g}$ pour l'ensemble de la partie non-grain 
pendant les périodes entre observations; ces différences seront négligées.

L'utilisation de réserves ainsi mesurée pourrait aussi être considérée comme une variation du stock de réserves. Cette méthode de différence peut être utilisée seulement parce que les grains constituent le seul organe-puits dans cette phase. Cette hypothèse, nettement démontrée par les courbes de croissance (non présentées ici), sera confirmée dans la suite du texte.

Cependant, la «mesure» de l'utilisation des réserves s'appuie sur des bilans de masse faits au rythme des pesées de matière sèche. Elle ne peut donc pas rendre compte d'échanges à une échelle d'espace ou de temps plus fine, c'est-à-dire d'échanges entre organes ou sur des périodes plus courtes que les intervalles entre observations.

\section{Principe de l'estimation de la croissance de plante entière à partir du rayonnement global disponible}

L'analyse énergétique de la fixation du carbone montre que l'efficience de la transformation du rayonnement intercepté en matière sèche peut être considérée globalement comme constante au cours du cycle de végétation, malgré quelques pics de courte durée lors de la mise en place d'organes nouveaux (Varlet Grancher, 1982). L'efficience biologique de la conversion de l'énergie solaire absorbée en matière sèche végétale est un rendement énergétique, c'est-à-dire le rapport entre l'énergie contenue dans la matière sèche végétale et l'énergie utilisée par le couvert pour sa fabrication (rayonnement intercepté $=\mathrm{Ri}$ ).

\section{Contenu énergétique}

Varlet Grancher (1982, et comm pers) montre que la valeur calorifique (ou contenu énergétique) de la matière sèche du maïs est assez stable au cours du cycle, (sauf aux périodes de fortes variations de teneurs en cendres, c'est-à-dire en début de cycle) et pour tous les organes de la plante, y compris les grains. Cette propriété permet d'exprimer l'efficience de conversion du rayonnement en matière sèche en g. $\mathrm{MJ}^{-1}$, avec la valeur employée habituellement aux autres périodes.

\section{Efficience de transformation du rayonnement en matière sèche}

L'efficience de la transformation est choisi à $3 \mathrm{~g} . \mathrm{MJ}^{-1}$ selon la valeur proposée par Bonhomme et al (1982). Elle sera vérifiée par comparaison des croissances de plante entière mesurées et des rayonnements interceptés calculés. Ce coefficient de transformation permet donc d'obtenir une estimation de la croissance du couvert à partir du rayonnement intercepté par la culture, lui-même calculé.

\section{Interception du rayonnement : calcul de l'énergie absorbée par le couvert}

Le rayonnement intercepté par la culture est estimé de façon théorique par l'expression proposée par Bonhomme et al (1982) pour le maïs :

$$
\Sigma R_{i}=0,46 \times \Sigma\left((1-\exp (-0,7 \times \text { indice foliaire })) R_{g}\right)
$$

où $-0,7$ est le coefficient calé expérimentalement par des mesures de rayonnement transmis sous un couvert de maïs, $R_{i}$ est le rayonnement intercepté par la culture, $R_{g}$ est le rayonnement global disponible pour la culture.

\section{RÉSULTATS}

\section{Évolution des quantités de matière sèche produites et utilisations de réserves}

La figure 1 représente l'évolution du poids de matière sèche de la plante entière et des grains ( $g$ /plante), pour un traitement d'un génotype ( $F 7$ $x$ F2, faible densité), présenté à titre d'exemple, sachant que tous les traitements ont des comportements analogues. Le lissage effectué en moyennant les valeurs sur 3 dates permet d'éliminer les irrégularités et, en particulier, les diminutions de matière sèche totale qui sont dues à la taille insuffisante des échantillons prélevés.

Les courbes d'utilisation de réserves (fig 1, bas) sont également moins accidentées. Notons

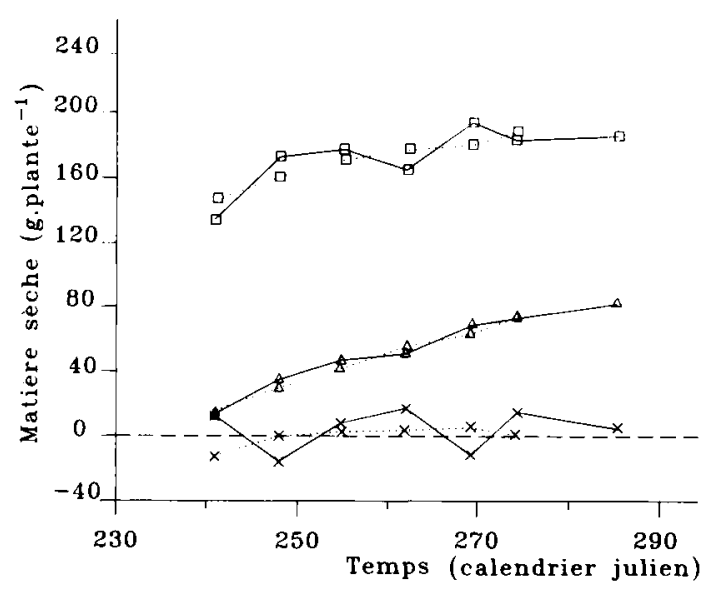

Fig 1. en haut : comparaison des courbes de croissance brutes et lissées (sur 3 dates) de plante entière et de grains; en bas : comparaison des mesures d'intervention des réserves à partir de courbes de croissance brutes ou lissées (génotype $\mathrm{F} 7 \times \mathrm{F} 2$, faible densité, floraison le jour 217,4 août). $\square$ plante entière, $\Delta$ grains; $x$ réserves; - mesures brutes; - - - mesures lissées. 
que les valeurs, quoique faibles, ne sont pas nulles, mais comprises entre 2 et $7 \mathrm{~g}$ par plante pour chaque période d'observation. Dans la suite du texte, les valeurs utilisées seront toujours celles des courbes lissées.

\section{Croissance de plante et appel aux réserves}

L'utilisation mesurée de réserves (croissance des grains - croissance de la plante entière) est inversement proportionnelle à la croissance de la plante entière (fig 2). Cette représentation fournit une explication de la régularité de croissance des grains et justifie l'emploi de cette loi en modélisation : les réserves interviennent pour compléter la croissance de la plante entière.

L'intersection de la droite de régression de l'ensemble de ces points avec l'axe des abscisses donne le niveau de croissance par plante à partir duquel il y a mise en réserves ou au contraire remobilisation. Ce seuil se situe entre 10,5 et $14,5 \mathrm{~g}$ (par plante pendant une semaine), ce qui correspond, selon les densités, à des rayonnements de 9-15 MJ.m-2.j-1. L'intersection de la droite de régression avec l'axe des ordonnées caractérise la demande des grains.

Ces 2 valeurs (seuil de mise en réserves et demande des grains) ne sont pas significativement différentes entre elles pour chaque traite-

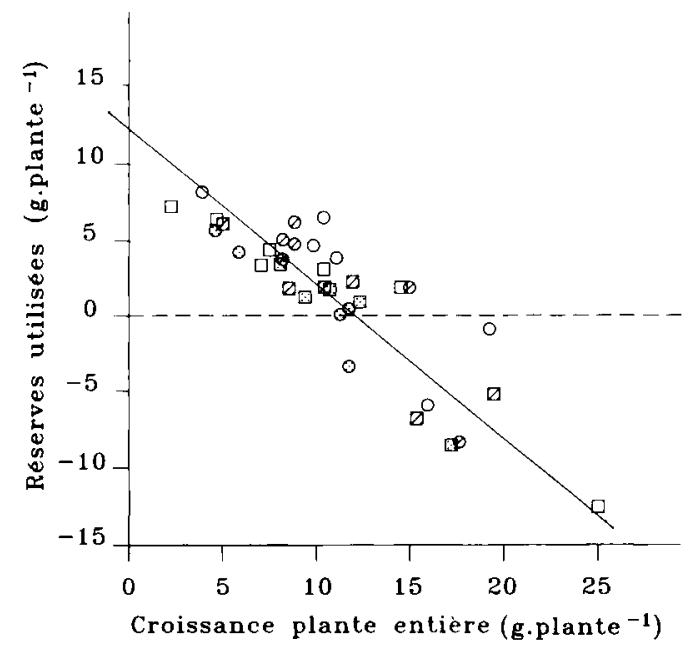

Fig 2. Relation entre utilisation de réserves et croissance de la plante entière sur la même période pour tous les traitements. $u=-1,01 c_{e}+12,36 ; r^{2}=0,76 ; u=$ utilisation de réserves; $c_{e}=$ croissance plante entière (g.plante ${ }^{-1}$. semaine ${ }^{-1}$ ). $\square$ faible densité; forte densité éclaircie; 圈forte densité; $\square$ F7 x F2; O Dea. ment : les grains constituent donc le seul puits à cette période, puisque les assimilats sont mis en réserves en dehors de la demande des grains.

\section{Évolution de l'utilisation des réserves}

Les cinétiques comparées d'utilisations de réserves (fig 3) montrent que celles-ci s'étalent sur une période longue pour tous les traitements, avec une tendance croissante qui reflète la diminution du rayonnement global : le rayonnement moyen passe de 14,5 en août (j 214-244) à 8,4 MJ.m-2.j-1 en septembre (j 245-274).

\section{Comparaison entre estimation et mesure des réserves utilisées}

L'ensemble des mesures d'utilisations de réserves a été comparé aux estimations correspondantes, pour chacun des intervalles entre observations (fig 4). La régression linéaire orthogonale (utilisée car la variable en abscisse est elle-même entachée d'erreur) donne :

$$
\begin{gathered}
\text { estimation }=(0,985 \text { mesure })+2,372 \quad r=0,556 \\
\left(\text { en } \mathrm{g} \cdot \mathrm{m}^{-2}, \text { pour des périodes d'une semaine }\right)
\end{gathered}
$$

La pente est proche de 1, l'ordonnée à l'origine proche de 0 , l'estimation est proche de la mesure, elle peut être considérée comme globalement satisfaisante. Le coefficient de corrélation relativement faible $\left(r^{2}=0,309\right)$ provient de points du début de remplissage de Dea (j 248-

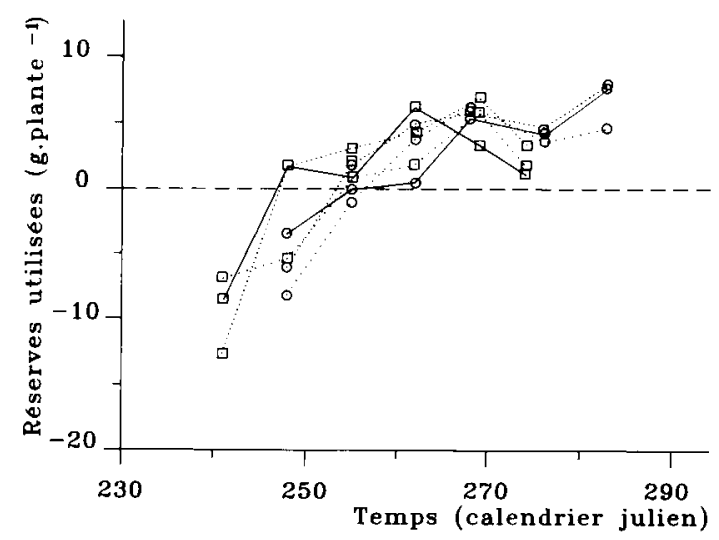

Fig 3. Évolution de l'utilisation des réserves pour le remplissage des grains, pour l'ensemble des traitements. - . faible densité; - - - forte densité éclaircie; - forte densité; F7 X F2, O Dea. 


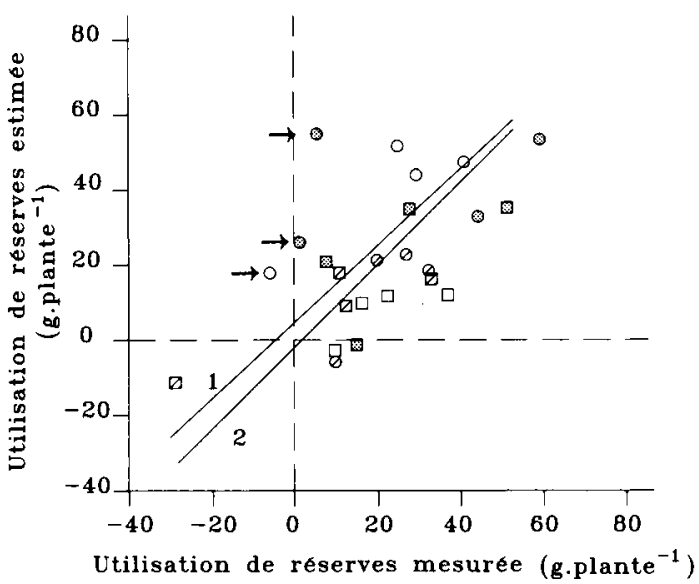

Fig 4. Comparaison entre mesures et estimations de l'intervention des réserves pour le remplissage des grains, au cours de périodes d'une semaine, pendant l'ensemble du remplissage. $\square$ faible densité; $\mathbb{Q}$ forte densité éclaircie; densité; 1 ajustement avec tous les points; 2 ajustements sans les points fléchés.

254 et 255-261 pour $d$ et $D$ ), où l'estimation des besoins en réserves est nettement supérieure à leur utilisation réelle. Cela signifierait que la disponibilité en assimilats pour la plante entière est sous-estimée par le modèle, autrement dit, que l'efficience de conversion du rayonnement intercepté est beaucoup plus forte que la valeur qui lui est attribuée dans le modèle.

Les efficiences de conversion du rayonnement calculées à partir des courbes de croissance de plante entière lissées (fig 5) montrent une valeur centrée autour de 3 , pour tous les traitements de F7 $\times$ F2. Pour Dea, au contraire, il apparaît un pic d'efficience au début de remplissage des grains. Ce pic correspondant à la mise en place d'un puits a déjà été signalé (Varlet Grancher, 1982), que ce soit au début de remplissage des grains ou à la période de croissance active de la tige.

L'existence d'un pic d'efficience de la conversion en début de remplissage fait que les estimations d'utilisation de réserves fondées sur une efficience constante ne sont pas applicables sur de courtes périodes situées en début de croissance active des grains.

Lorsque les points de début de remplissage, correspondant au pic d'efficience, ne sont pas pris en compte, la relation entre valeurs estimées et mesurées devient :

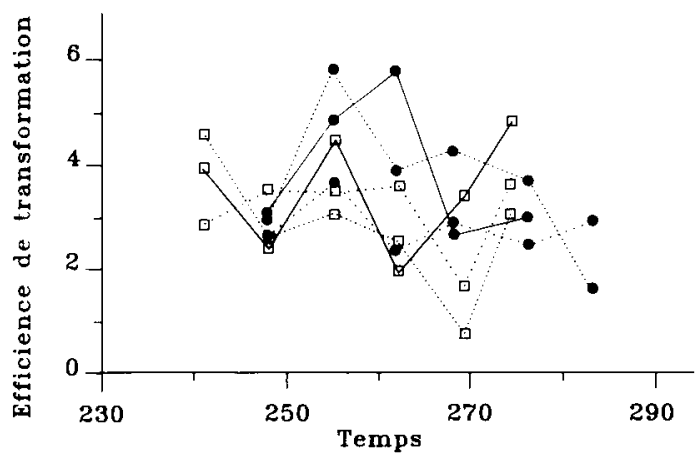

Fig 5. Évolution de l'efficience de conversion du rayonnement intercepté pendant le remplissage des grains, pour tous les traitements. - . faible densité; - - - forte densité éclaircie; - forte densité; $\square$ F7 × F2, O Dea.

estimation $=1,008 \times$ mesure $-2,587 \quad r=0,738$

Les paramètres de la droite sont peu modifiés, mais la quatité de la régression est nettement améliorée $\left(r^{2}=0,545\right)$.

L'estimation faite ici n'est pas précise (limites de confiance de l'estimation d'une moyenne conditionnelle situées entre 6 et $10 \mathrm{~g}$ par plante, dans la gamme des valeurs observées). Son objectif est plutôt de donner une indication des périodes où il doit $y$ avoir utilisation de réserves, que la quantification précise de celles-ci. II faut noter qu'une partie de l'imprécision provient de la mesure de remobilisation faite à partir de courbes de croissance en matière sèche insuffisamment précises (population non suffisamment homogène, échantillon trop petit).

La méthode proposée permet d'estimer les périodes d'intervention des réserves et, éventuellement, leur quantité, en dehors du début de remplissage actif des grains.

Elle peut donc être utilisée pour construire un protocole expérimental permettant de provoquer l'utilisation des réserves au moment où sont prévus des dosages ou des tests de transfert d'assimilats. Cette méthode permet aussi de se placer dans des conditions de recours aux réserves qui permettent d'épuiser celles-ci pour mesurer ensuite les matières sèches restantes qui représentent les quantités de structures fabriquées.

Cet outil de caractérisation de l'utilisation de réserves apparaît donc comme un complément maniable de méthodes de dosages plus difficiles à mettre en œuvre. 


\section{CONCLUSION}

La disponibilité en assimilats d'un couvert peut donc être estimée à partir du rayonnement intercepté par la culture, si l'on se place en dehors des forts pics d'efficience de conversion du rayonnement de début de remplissage actif des grains. Cette méthode permet d'estimer les quantités de réserves utilisées lors du remplissage des grains. Elle ne s'appuie que sur des mesures simples : évolution du poids des grains, indice foliaire et rayonnement global disponible à la même période. Elle est évidemment applicable à d'autres plantes remplissant un organe unique, avec complément de réserves temporaires lorsque les sources directes d'assimilats sont insuffisantes.

\section{RÉFÉRENCES}

Barnett KH, Pearce RB (1983) Source-sink ratio alteration and its effect on physiological parameters in maize. Crop Sci 23, 294-299

Bonhomme R, Ruget F, Derieux M, Vincourt P (1982) Relations entre production de matière sèche aérienne et énergie interceptée chez différents génotypes de maïs. CR Séances Acad Sci Paris Ser III 294, 393-398

Cliquet JB (1989) Étude de la mobilisation du carbone et de l'azote des parties végétatives vers le grain chez le maïs, effet d'un raccourcisseur de tige, l'étéphon. Thèse de Doctorat, Biol Physiol Vég, Univ Paris VI, $58 \mathrm{p}$

Daynard TB, Tanner JW, Hume DJ (1969) Contribution of stalk soluble carbohydrates to grain yield in corn (Zea mays L). Crop Sci 9, 831-834

Derieux M (1978) Étude de quelques facteurs d'adaptation du Maïs aux conditions climatiques du Nord de la France. Ann Amelior Plant 28, 529-566

Egharevba PN, Horrocks RD, Zuber MS (1976) Dry matter accumulation in maize in response to defoliation. Agron J 68, 40-43

Iwata F (1975) Ear barreness of corn as affected by plant population. JARQ 9, 13-17

Johnson DR, Tanner JW (1972) Calculation of the rate and duration of grain filling in corn (Zea mays L) Crop Sci 12, 485-486

Jones CA, Kiniry JR (1986) CERES-maize, a simulation model of maize growth and development. Texas A \& M Univ

Jones RJ, Simmons SR (1983) Effect of altered source-sink ratio on growth of maize kernels. Crop Sci $23,129-134$

Kiniry JR, Ritchie JT (1985) Shade-sensitive interval of kernel number of maize. Agron J 77, 711-715

Ruget $F$ (1989) Relations entre matière sèche à la floraison et rendement en grains chez le maïs : importance du rayonnement disponible par plante. Agronomie 9, 457-465

Varlet Grancher C (1982) Analyse du rendement de la conversion de l'énergie solaire par un couvert végétal. Thèse Docteur ès Sciences Naturelles, univ Paris-Sud, centre d'Orsay, $144 \mathrm{p}$ 